\title{
The Design and Realization of Personalized E-commerce Recommendation System
}

\author{
Xiaosen WEN \\ Xijing University,Xi'an,Shaanxi,710123 China
}

\begin{abstract}
This paper analyze and design the requirements and function of personalized e-commerce recommendation from the electronic commerce recommendation system needs, and distinguished on the system from the macro level, design and complete different system functions in a different system level; then detailed design the system process and the key data in the database from the front desk system and the background system. Finally, this paper simply test respectively on mainly model of the front and back systems.

KEYWORD: Personalized Recommendation; E-commerce; Recommended System;
\end{abstract}

\section{INTRODUCTION}

With the expansion of the scale of e-commerce, it provides a richer choice for users. However, for the face of such diverse commodity information, how can fast and accurately pick up the goods they need has become the topic of most concern to users. In this context, ecommerce recommendation system emerge. ecommerce recommendation system grasp the key data from the rich data information, mining potential customers for the business, expanding the scope of sales, but also for the old customers to provide product recommendation, enlarge an user group. For users, electronic commerce recommendation system can be personalized recommendation based on user preferences, interest and other characteristics, a user can quickly accurately select the goods they need in the huge commodity information, reduce the waste in the commodity selection process in time, to provide users with more humanized service. Therefore, business or the user has huge development prospect and application value of electronic commerce recommendation system.

\section{Personalized Recommendation System MODEL}

Personalized recommendation system, the key is to store the user interested information. In general, the personalized recommendation system can be divided into four levels: data collection, user modeling, algorithm of recommendation, recommend output. Personalized recommendation system model is as shown in figure 1 .

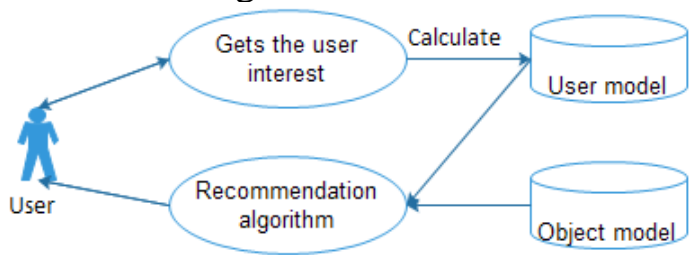

Fig.1 Personalized recommendation system model

(1) Data collection

Data collection is the premise and basis to establish the personalized recommendation system model. Collection of data were the process that obtain the user information, preferences, characteristics and interest data. The data collection process mainly have in three ways: explicit acquisition, implicit access and heuristic to obtain.

(2) User modeling

In order to accurately reflect the various users, dynamic change of interest, we need to establish a user model for user. The process of user modeling is as shown in figure 2 .

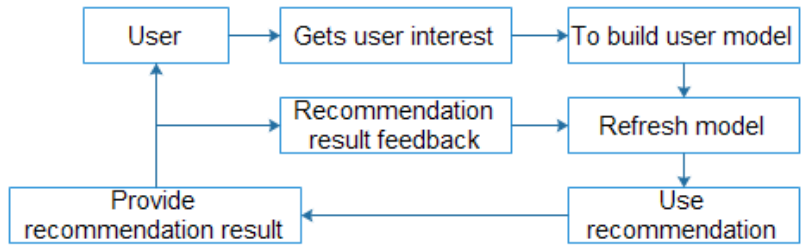

Fig.2 User modeling process

(3) Recommendation algorithm

Recommendation algorithm determines the personalized recommendation system types, properties and quality fundamentally. Recommendation algorithm can establish user model according to the collected user information, learning algorithm by setting goals 
to calculate the specific user recommendation results.

\section{COMPOSITION OF E-COMMERCE RECOMMENDATION SYSTEM}

The overall structure of the system can be divided into three parts for e-commerce recommendation: input module, recommendation output module and the recommendation algorithm module. The relationship between the various modules is as shown in Figure 3.

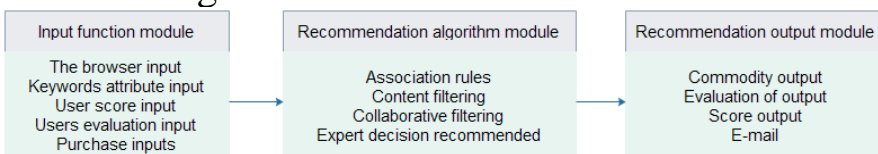

Fig.3 Composition of e-commerce recommendation system

\subsection{The system architecture design}

According to the demand analysis of ecommerce recommendation system based on personalized recommendation, the system architecture can be divided into three levels: the foreground interactive layer, business logic layer, data service layer. System hierarchy is as shown in Figure 4

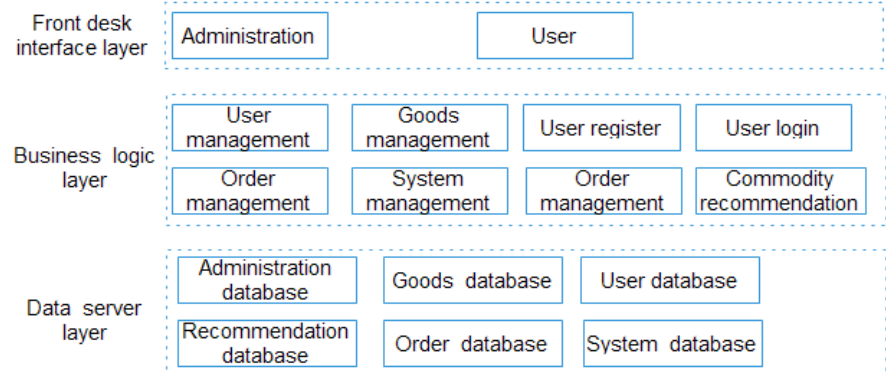

Fig.4 The system hierarchy diagram

The front desk interactive layer is interface of the user and administrator that interact with the system, all interaction system users and the system is completed by the front desk interaction layer. The system user interaction layer sends instructions to the system for processing through the front and the corresponding operation to complete the processing of the system, the results will be returned to the foreground interactive layer, presentation to the users.

\subsection{The front desk system flow}

The front desk system oriented the main users of the web site, so the foreground system needs to provide a simple and friendly interface for user, also need to provide all the functions of electronic commerce system for users. The front desk system flow chart is as shown in figure 5.

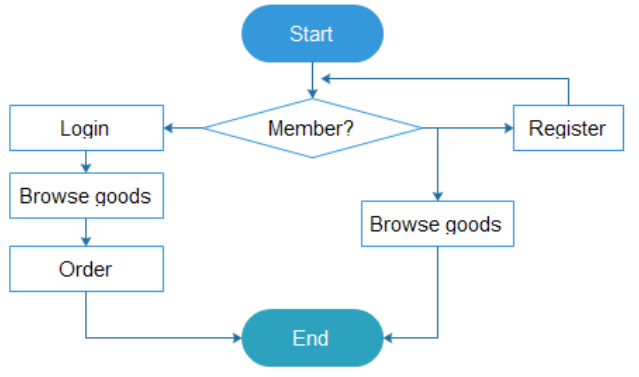

Fig.5 The flow chart of front desk system

\subsection{The recommended procedure}

E-commerce personalized recommendation system can respectively recommend goods for new users, registered users and users of old. For new users, the system will recommend currently the best-selling merchandise for the user, are selling goods recommendation. For registered users, information providing system has record users age, occupation, interests, hobbies and so on, therefore to purchase goods for the user can be interested the commodity recommendation based on user characteristics. The old users buy more goods through the website, system has record user more shopping information. So the system can calculate the user preferences, also can according to user requirements for user preference goods recommendation. The recommended procedure is shown in figure 6.

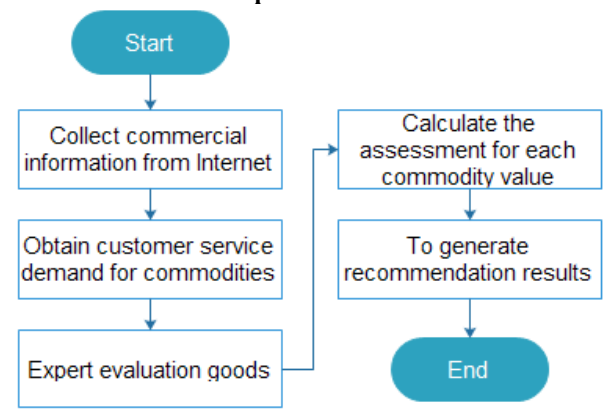

Fig.6 Recommended flow chart of expert decision system 


\section{EXPERT DECISION RECOMMENDATION ALGORITHM}

Expert knowledge analysis module obtain the quantitative functional feature vector attributes of goods $F=\left\langle f_{1}, f_{2}, \ldots, f_{n}\right\rangle$, and have user demand vector $W=\left\langle\omega_{1}, \omega_{2}, \ldots, \omega_{n}\right\rangle$.So, the attribute of commodity, commodity property is in according with customer demand description. Analysis of the specific algorithm is as follows: (1). According to the commodity attribute index, to establish decision A for initial matrix commodity information:

$$
A=\left(\begin{array}{llll}
a_{11} & a_{12} & \ldots & a_{1 n} \\
a_{21} & a_{22} & \ldots & a_{2 n} \\
\vdots & \vdots & & \vdots \\
a_{m 1} & a_{m 2} & \ldots & a_{m n}
\end{array}\right)=\left(\mathbf{a}_{i j}\right)_{m \times n}
$$

aij is the No. j property value of NO. i product.

(2). Standardize the initial decision matrix $A^{\prime}=\left(\mathrm{a}_{i j}^{\prime}\right)_{m \times n}$

(3). Standardize the demand matrix of user $W=\left\langle\omega_{1}, \omega_{2}, \ldots, \omega_{n}\right\rangle$, and get customer demand

vector $W^{\prime}=\left\langle\omega_{1}^{\prime}, \omega_{2}^{\prime}, \ldots, \omega_{n}^{\prime}\right\rangle$ normalization

(4). to determine the positive ideal solution and the negative ideal solution.

$$
\begin{aligned}
& \mathrm{A}^{+}=\left\{\left(\max _{1 \leq i \leq m} a_{i j} \mid j \in J^{+}\right),\left(\min _{1 \leq i \leq m} a_{i j} \mid j \in J^{-}\right)\right\}=\left\{a_{1}^{+}, a_{2}^{+}, \cdots, a_{n}^{+}\right\} \\
& \mathrm{A}^{-}=\left\{\left(\min _{1 \leq i \leq m} a_{i j} \mid j \in J^{+}\right),\left(\max _{1 \leq i \leq m} a_{i j} \mid j \in J^{-}\right)\right\}=\left\{a_{1}^{-}, a_{2}^{-}, \cdots, a_{n}^{-}\right\}
\end{aligned}
$$

$\mathrm{J}+$ is the income attribute, indicates the optimal value of No.i properties; J - is loss type attribute, means the worst value of No.i attributes.

(5). For each commodity in the library that will be select goods, calculate their respective the positive, negative ideal solution distance.

$$
\begin{aligned}
& S^{+}=\sqrt{\sum_{j=1}^{n}\left[\omega_{j}^{\prime}\left(a_{i j}^{\prime}-a_{j_{-} \text {best }}^{\prime}\right)\right]^{2}} \\
& S^{-}=\sqrt{\sum_{j=1}^{n}\left[\omega_{j}^{\prime}\left(a_{i j}^{\prime}-a_{j_{-} \text {worst }}^{\prime}\right)\right]^{2}}
\end{aligned}
$$

(6). the utility function calculate the utility value that will be select goods

$$
U_{i}=\frac{S_{i}^{-}}{S_{i}^{+}+S_{i}^{-}}
$$

The utility value represents the closeness to be the selection of goods and the ideal solution, the higher the utility value of goods, and it is more close to ideal solution. After computing all selected commodity utility value, the maximum value is the best recommendation.

\section{EXPERIMENTAL TESTING OF ALGORITHM MODEL}

Two users need to buy a computer, the experimental input user demand data, through a series of calculation steps in expert decision algorithm model, to sort the goods stored in the current database, and get the most similar recommended goods need by the user, the input data are shown in table 1.

Table.1 User requirements data

\begin{tabular}{c|c|c|c|c|c}
\hline Performance & CPU & Memory & Graphics card & Battery & Price \\
\hline User 1 & 7 & 7 & 5 & 8 & 6 \\
\hline User 2 & 8 & 6 & 8 & 5 & 5
\end{tabular}

Expert decision algorithm recommendation model first standardize the commodity information stored in the database and so on a series of processing, obtains the standard matrix normalized, see Table 2 and 3.

Table. 2 The original commodity information matrix

\begin{tabular}{l|l|l|l|l}
\hline 7 & 7 & 5 & 8 & 6 \\
\hline 8 & 5 & 6 & 5 & 6 \\
\hline 9 & 8 & 9 & 9 & 8 \\
\hline 6 & 5 & 5 & 6 & 4 \\
\hline 5 & 8 & 5 & 6 & 7 \\
\hline 6 & 8 & 7 & 7 & 7 \\
\hline
\end{tabular}

Table. 3 the standardization commodity information matrix

\begin{tabular}{l|l|l|l|l}
\hline 0.410 & 0.410 & 0.334 & 0.468 & 0.379 \\
\hline 0.468 & 0.334 & 0.379 & 0.334 & 0.379 \\
\hline 0.527 & 0.468 & 0.527 & 0.527 & 0.468 \\
\hline 0.379 & 0.334 & 0.334 & 0.379 & 0.252 \\
\hline 0.334 & 0.468 & 0.334 & 0.379 & 0.410 \\
\hline 0.379 & 0.468 & 0.410 & 0.410 & 0.410 \\
\hline
\end{tabular}

Tables 4 and 5 were given test data of the positive, negative ideal distance solution, the utility value and the recommendation results sorting for corresponding goods. 
Table.4 User1 data test results

\begin{tabular}{l|l|l|l|c}
\hline \multicolumn{1}{c|}{ N\A } & \multicolumn{1}{|c|}{$\mathrm{S}+$} & \multicolumn{1}{|c|}{$\mathrm{S}-$} & \multicolumn{1}{c|}{$\mathrm{E}$} & Sequence \\
\hline Optional goods 1 & 0.277 & 0.216 & 0.438 & 2 \\
\hline Optional goods 2 & 0.351 & 0.187 & 0.348 & 3 \\
\hline Optional goods 3 & 0.0 & 0.0 & 0.0 & 6 \\
\hline Optional goods 4 & 0.443 & 0.143 & 0.244 & 5 \\
\hline Optional goods 5 & 0.360 & 0.149 & 0.292 & 4 \\
\hline Optional goods 6 & 0.230 & 0.212 & 0.479 & 1 \\
\hline
\end{tabular}

Table.5 User1 data test results

\begin{tabular}{c|c|c|c|c}
\hline N\A & S+ & S- & E & Sequence \\
\hline Optional goods 1 & 0.221 & 0.232 & 0.513 & 1 \\
\hline Optional goods 2 & 0.336 & 0.187 & 0.357 & 4 \\
\hline Optional goods 3 & 0.437 & 0.125 & 0.222 & 6 \\
\hline Optional goods 4 & 0.261 & 0.239 & 0.478 & 3 \\
\hline Optional goods 5 & 0.385 & 0.164 & 0.299 & 5 \\
\hline Optional goods 6 & 0.370 & 0.212 & 0.481 & 2 \\
\hline
\end{tabular}

\section{Conclusions}

From the above two test table data can be seen, for the different users data, through the expert recommended decision model provide most consistent with the user demand goods in the optional commodity list system, and as the results presented to the user recommendation. This paper presents a series of test data and the results of experts recommended decision algorithm model, in order to validate the feasibility of the recommended model.

\section{REFERENCE}

[1] Zhao Xiaohong. The main mode of the future of ecommerce B2B [J]. Journal of Southwest Nationalities College, 2001, 11:1-3.

[2] Li Ziyao. Innovation strategy of B2C e-commerce mode [J]. Commercial age, 2008, 12:88.

[3] Tang Guanglin, Wu Yong, Meng Weidong. Compared with typical B2C e-commerce website [J]. Modern mall, 2007, 12: 77.

[4] Xu Yulin, Zhou Lei. The C2C electronic commerce website profit way [J]. Computer world, 2009,1-7.

[5] Liu Jianguo, Zhou Tao, Guo Qiang, Wang Binghong. Personalized recommendation system evaluation method summary $[\mathrm{J}]$. Complex systems and complexity science, 2009, 6 (3): 1-10. 